\title{
Variational Principles of Fuzzy Mappings and Its Applications
}

\author{
Yu-E Bao*, Ying-Chun Niu, Yuan Li \\ College of Mathematics, Inner Mongolia University for Nationalities, Inner Mongolia Tongliao, P. R. China
}

\author{
Email address: \\ byebed@163.com (Yu-E Bao) \\ ${ }^{*}$ Corresponding author
}

\section{To cite this article:}

Yu-E Bao, Ying-Chun Niu, Yuan Li. Variational Principles of Fuzzy Mappings and Its Applications. Science Journal of Applied Mathematics and Statistics. Vol. 5, No. 4, 2017, pp. 139-146. doi: 10.11648/j.sjams.20170504.13

Received: June 3, 2017; Accepted: July 5, 2017; Published: July 19, 2017

\begin{abstract}
In this paper, we firstly discuss the basic properties of the sub differential of fuzzy mapping and get some related conclusions. Secondly, we establish a variational principle of fuzzy mapping by establishing the concept of gauge fuzzy mapping. Then we prove the approximation sun rule of fuzzy mapping in sub-differential as the application of that principles.
\end{abstract}

Keywords: Fuzzy Mapping, Sub-differential, Gauge Mapping, Variational Principle, Approximation Sun Rule

\section{Introduction}

In 1972, Chang and Zadeh [1] introduced the concept of fuzzy numbers with the consideration of the properties of probability functions. Since then the fuzzy numbers and fuzzy mapping of the value is fuzzy number have been extensively studied by many authors. Researchers have begun to study upon the relation equations of fuzzy numbers, the differential and measurability and integral of fuzzy mappings, and other theories in relation to fuzzy numbers. Especially in the differentiability of fuzzy mapping and its application in fuzzy programming, a series of important achievements have been reached [2, 3, 4]. However, not all of the fuzzy mapping is differentiable. So we should also study those fuzzy mappings whose analytic properties are weaker than differentiability. Concept of sub-differential of convex fuzzy mapping is introduced in [5, 6]. Its related properties and application in convex programming is discussed and some important conclusions are obtained.

In $[7,8]$ we extend the concept of sub-differential of convex fuzzy mapping given in $[5,6]$, establish the concept of sub-differential of normal fuzzy mapping and study the existence problem of the convex extension of fuzzy mapping. New study method is provided to search on fuzzy programming problems. By using the study methods and techniques of non-smooth analysis and set valued analysis [9,
$10,11]$, we discuss the basic properties of sub-differential of fuzzy mapping [6] and try to establish a variational principle of fuzzy mapping. Finally we discuss the application of it.

\section{Prerequisite}

Let $R$ be the real numbers field A fuzzy set $u$ on $R$ is called a fuzzy number, if it has the following properties:

(I) $u$ is upper semi-continuous;

(II) $u$ is normal, i.e., there exists an $x_{0} \in R$ such that $u\left(x_{0}\right)=1$;

(III) $u$ is convex, i.e.,

$$
u(\lambda x+(1-\lambda) y) \geq \min (u(x), u(y))
$$

Whenever $x, y \in R, r \in[0,1]$.

(IV) $[u]^{0}=\overline{\{x \mid u(x)>0\}}$ is a compact set.

Let $\mathcal{F}$ denote the family of all fuzzy numbers and is called fuzzy number space. See the concepts and its properties related to fuzzy number in [4]. For any $r \in R$, we define a fuzzy number $\tilde{r}$ by

$$
\tilde{r}(t)=\left\{\begin{array}{l}
1, t=r \\
0, t \neq r
\end{array}\right.
$$

for any $t \in R$. Let $\tilde{R}=\{\tilde{r} \mid r \in R\}$, then $\tilde{R} \subset \mathcal{F}$. For 
$u \in \mathcal{F}, \alpha \in[0,1]$, the r-level set of fuzzy number $u$ is a nonempty bounded closed interval, which is denoted as

$$
[u]^{\alpha}=\left[u_{*}(\alpha), u^{*}(\alpha)\right] .
$$

For $u, v \in \mathcal{F}$, we say that $u \leq v$ if and only if

$$
u^{*}(\alpha) \leq v^{*}(\alpha) \text { and } u_{*}(\alpha) \leq v_{*}(\alpha)
$$

for any $\alpha \in[0,1]$.

If $u \geq 0$, then $u$ is called a nonnegative fuzzy number, and $\mathcal{F}_{+}$denote the family of all nonnegative numbers.

For $u, v \in \mathcal{F}$ and $r \in R$, we define the addition $u+v$ and scalar multiplication $r u$ as follows:

$$
[u+v](\alpha)=\left[u_{*}(\alpha)+v_{*}(\alpha), u^{*}(\alpha)+v^{*}(\alpha)\right],
$$

for any $\alpha \in[0,1]$.

If $r \geq 0$,

$$
[r u](\alpha)=\left[r u_{*}(\alpha), r u^{*}(\alpha)\right]
$$

for any $\alpha \in[0,1]$.

$$
[r u](\alpha)=\left[r u^{*}(\alpha), r u_{*}(\alpha)\right]
$$

for any $\alpha \in[0,1]$.

For $\xi_{i}(i=1,2, \cdots, n) \in \mathcal{F}$, we call

$$
\xi=\left(\xi_{1}, \cdots, \xi_{n}\right)
$$

is an $n$-dimensional fuzzy vector. The set of all $n$ -dimensional fuzzy vectors is denoted by $\mathcal{F}^{n}(\mathrm{R})$. For

$$
\begin{aligned}
& \xi=\left(\xi_{1}, \xi_{2}, \cdots, \xi_{n}\right) \in \mathcal{F}^{n}(\mathrm{R}) \\
& x=\left(x_{1}, x_{2}, \cdots, x_{n}\right) \in \mathrm{R}^{n}
\end{aligned}
$$

the inner product of $\xi$ and $x$ is defined as

$$
\begin{aligned}
& \xi=\left(\xi_{1}, \xi_{2}, \cdots, \xi_{n}\right) \in \mathcal{F}^{n}(\mathrm{R}) \\
& x=\left(x_{1}, x_{2}, \cdots, x_{n}\right) \in \mathrm{R}^{n}
\end{aligned}
$$

the inner product of $\xi$ and $x$ is defined as

$$
\langle\xi, x\rangle=x_{1} \xi_{1}+x_{2} \xi_{2}+\cdots+x_{n} \xi_{n}
$$

If for $u, v \in \mathcal{F}$, there exists $w \in \mathcal{F}$ such that $u=v+w$, we say that the H-difference of $u$ and $v$ exist and denote $u-v=w$.

It is obvious that if H-difference $u-v$ exists, then

$$
\begin{aligned}
& (u-v)_{*}(\alpha)=u_{*}(\alpha)-v_{*}(\alpha) \\
& (u-v)^{*}(\alpha)=u^{*}(\alpha)-v^{*}(\alpha)
\end{aligned}
$$

Definition 2.1 [12]. Let $S$ be a subset of $\mathcal{F}$. If there exists $u_{0} \in \mathcal{F}$ such that $u \geq u_{0}$ for any $u \in S$, then $u_{0}$ is called the lower bounded of $S$.

If $u_{0}$ is a lower bounded of $S$, and satisfies that for any lower bound $u^{\prime}$ of $S u_{0} \leq u^{\prime}$. Then $u_{0}$ is called the infimum of $S$ and denote as $\inf S=u_{0}$.

A upper bound and the supermun of $S$ are defined similarly. $S$ is said to be order bounded if it is both bounded from above and bounded from below.

Let $M$ be a nonempty sub set of n-dimensional Euclidean space $R^{n}$. We call the mapping from $M$ to $\mathcal{F}$ a fuzzy mapping (fuzzy number value function), and denote as $F: M \rightarrow \mathcal{F}$. For any $\alpha \in[0,1]$, denote

$$
[F(x)](\alpha)=\left[F_{*}(x)(\alpha), F^{*}(x)(\alpha)\right] \text {, }
$$

where $F_{*}(x)(\alpha)$ and $F^{*}(x)(\alpha)$ are real value function defined in $M$.

Definition 2.2 [13] Let $F: M \rightarrow \mathcal{F}$ be a fuzzy mapping, $x_{0} \in M . F$ is lower semi-continuous at a point $x_{0}$ If for any $\varepsilon>0$, there exists $\delta>0$ such that

$$
F\left(x_{0}\right) \leq F(x)+\tilde{\varepsilon} .
$$

For all $x \in M$ and $\left\|x-x_{0}\right\|<\delta$. Then we say that $F$ is lower semi-continuous at each point of $M$.

Theorem 2.1. Let $F: M \rightarrow \mathcal{F}$ be a fuzzy mapping. If $F$ is a lower semi-continuous, $u \in \mathcal{F}$, then

$$
A^{u}(F)=\{x \in M \mid F(x) \leq u\}
$$

is closed set in $R^{n}$.

Proof. for $u \in \mathcal{F}$, we have

$$
\begin{gathered}
F(x) \leq u \\
\Leftrightarrow F(x)_{*}(\alpha) \leq u_{*}(\alpha) \text { and } F(x)^{*}(\alpha) \leq u^{*}(\alpha)
\end{gathered}
$$

for any $\alpha \in[0,1]$.

Therefore,

$$
\begin{aligned}
A^{u}(F)= & \left\{x \in M \mid F(x)_{*}(\alpha) \leq u_{*}(\alpha), \alpha \in[0,1]\right\} \\
& \bigcap\left\{x \in M \mid F(x)^{*}(\alpha) \leq u^{*}(\alpha), \alpha \in[0,1]\right\}
\end{aligned}
$$

On the other hand, by $F: M \rightarrow \mathcal{F}$ is semi-continuity, we know that for any $\alpha \in[0,1]$, both $F(x)_{*}(\alpha)$ and $F(x)^{*}(\alpha)$ are lower semi-continuous real valued functions 
defined in $M$.

So for any $\alpha \in[0,1]$, we have

$$
\left\{x \in M \mid F(x)_{*}(\alpha) \leq u_{*}(\alpha)\right\}
$$

and

$$
\left\{x \in M \mid F(x)^{*}(\alpha) \leq u^{*}(\alpha)\right\}
$$

are closed set. Hence $A^{u}(F)$ is closed set.

Definition 2.3 [6]. Let $F: M \rightarrow \mathcal{F}$ be a fuzzy mapping, $x \in M$. The sub-differential o $F$ f at $x$ is defined as $\partial F(x)$

$=\left\{\xi \mid \xi \in \mathcal{F}^{n}(\mathrm{R}), F(z) \geq F(x)+\langle\xi, z-x\rangle, \forall z \in M\right\}$

If $\partial F(x) \neq \varnothing$, then $F$ is called sub-differentiable at $x$.

If $\partial F$ is a single point set $\{\xi\}$, then $F$ is called differentiable at $x$, and denote as $\xi=\nabla F(x)$.

Theorem 2.2 . Let $F, F_{i}: M \rightarrow \mathcal{F}(i=1,2)$ be fuzzy mappings, $x \in M$, then

(I) $\partial(\lambda F)(x)=\lambda F(x)(\lambda>0)$

(II) $\partial\left(F_{1}+F_{2}\right)(x) \supset \partial F_{1}(x)+\partial_{2}(x)$.

Proof. (I) For

$$
\xi=\left(u_{1}, u_{2}, \cdots, u_{n}\right) \in \partial(\lambda F)(x),
$$

by Definition 2.3, we have

$$
\lambda F(z) \geq \lambda F(x)+\langle\xi, z-x\rangle
$$

for any $z \in M$.

Therefore

$$
F(z) \geq F(x)+\left\langle\frac{1}{\lambda} \xi, z-x\right\rangle
$$

So

$$
\frac{1}{\lambda} \xi \in \partial F(x)
$$

i.e., $\xi \in \lambda \partial F(x)$.

Therefore

$$
\partial(\lambda F)(x) \subset \lambda \partial F(x) .
$$

We can similarly obtain

$$
\lambda \partial F(x) \subset \partial(\lambda F)(x) .
$$

So

$$
\lambda \partial F(x)=\partial(\lambda F)(x)
$$

(II) Let

$$
\xi=\left(u_{1}, u_{2}, \cdots, u_{n}\right) \in \partial F_{1}(x)+\partial F_{2}(x),
$$

then there exist $\xi_{1} \in \partial F_{1}(x), \xi_{2} \in \partial F_{2}(x)$ such that

$$
\xi=\xi_{1}+\xi_{2}
$$

and by Definition 2.3, we have

$$
\begin{aligned}
& F_{1}(z) \geq F_{1}(x)+\left\langle\xi_{1}, z-x\right\rangle \\
& F_{2}(z) \geq F_{2}(x)+\left\langle\xi_{2}, z-x\right\rangle
\end{aligned}
$$

for any $z \in M$.

According to (1)+(2), we have

$$
\left(F_{1}+F_{2}\right)(z) \geq\left(F_{1}+F_{2}\right)(x)+\left\langle\xi_{1}+\xi_{2}, z-x\right\rangle .
$$

So

$$
\xi=\xi_{1}+\xi_{2} \in \partial\left(F_{1}+F_{2}\right)(x),
$$

i.e.,

$$
\partial F_{1}(x)+\partial F_{2}(x) \subset \partial\left(F_{1}+F_{2}\right)(x) .
$$

We can easily obtain the following Corollary 2.1 according to Theorem 2.2.

Corollary 2.1. Let $F_{i}: M \rightarrow \mathcal{F}(i=1,2)$ be fuzzy mapping, and

$$
F_{1}(x)=\lambda F_{2}(x)(\lambda>0)
$$

for any $x \in M$, then

$$
\partial F_{1}(x)+\partial F_{2}(x)=\partial\left(F_{1}+F_{2}\right)(x)
$$

Theorem 2.3. Let $F: M \rightarrow \mathcal{F}$ be fuzzy mapping. If $F\left(x_{0}\right)$ is the minimum of $F$ in $M$, then

$$
0 \in \partial F\left(x_{0}\right)(0=(\hat{0}, \hat{0}, \cdots, \hat{0},)) .
$$

Proof. Let $F\left(x_{0}\right)$ be the minimum of $F$ in $M$, then for any $x \in M$,

$$
F(z) \geq F\left(x_{0}\right)
$$

Therefore, for any $z \in M$ and $0=(\hat{0}, \hat{0}, \cdots, \hat{0}$,$) , we have$

$$
F(z) \geq F\left(x_{0}\right)+\left\langle 0, z-x_{0}\right\rangle
$$

i.e., $0 \in \partial F\left(x_{0}\right)$. 


\section{Fuzzy Variational Principles}

$X$ stands for $R^{n}$ or a sub set of $R^{n}$ in this section.

Definition 3.1. Let $(X, d)$ be complete metric space, continuous mapping $\rho: \mathrm{X} \times X \rightarrow \mathcal{F}_{+}$is called a gauge fuzzy mapping. If it has the following properties:

(I) $\rho(x, x)=\hat{0}, \forall x \in X$;

(II) For arbitrary $\varepsilon>0$, there exists $\delta>0$, such that

$$
d(y, z)<\varepsilon
$$

for any $y, z \in X$ when $\rho(y, z) \leq \hat{\delta}$;

(III) $\rho(X \times X)$ is the totally ordered sub set of $\mathcal{F}_{+}$.

Theorem 3.1 (Variational Principles of Fuzzy Mapping). Let $(X, d)$ be a complete metric space. If fuzzy mapping $F: M \rightarrow \mathcal{F}$ satisfy conditions (I)-(III):

(I) $F(X)$ is a totally ordered sub set of $\mathcal{F}$ with a H-difference;

(II) $F$ is lower semi-continuous and $\inf _{X} F \in F(X)$;

(III) $\rho$ is a gauge fuzzy mapping, $\left\{\delta_{n}\right\}_{n=0}^{\infty}$ is a positive sequence of points.

Then for any $\varepsilon>0$ and $z \in X$, when

$$
F(z) \leq \inf _{X} F+\hat{\varepsilon}
$$

there exists a sequence of points $x_{n} \in X(n=1,2, \ldots)$ such that $x_{n} \rightarrow x(n \rightarrow \infty)$ and

(I) $\rho(z, y) \leq \tilde{\varepsilon} / \sigma_{0}$

when $F(z) \leq \inf _{X} F+\hat{\varepsilon}$;

(II) $F(y)+\sum_{n=0}^{\infty} \sigma_{n} \rho\left(y, x_{n}\right) \leq F(z)$

when $F(z) \leq \inf _{x} F+\hat{\varepsilon}$;

(III) $F(x)+\sum_{n=0}^{\infty} \sigma_{n} \rho\left(x, x_{n}\right) \geq F(y)+\sum_{n=0}^{\infty} \sigma_{n} \rho\left(y, x_{n}\right)$ for any $x \in X$.

Proof. First we build a sequence of points $\left\{x_{n}\right\}$ which satisfies $x_{n} \in X(n=1,2, \ldots)$ and $x_{n} \rightarrow x(n \rightarrow \infty)$.

Let

$$
\begin{gathered}
x_{0}=z, \\
S_{0}=\left\{x \in X \mid F(x)+\sigma_{0} \rho\left(x, x_{0}\right) \leq F\left(x_{0}\right)\right\}
\end{gathered}
$$

Because $F$ and $\rho\left(\cdot, x_{0}\right)$ are lower semi-continuous, $S_{0}$ is closed set according to Theorem 2.1. And for any $x \in S_{0}$, we have

$$
\begin{aligned}
\sigma_{0} \rho\left(x, x_{0}\right) & \leq F\left(x_{0}\right)-F(x) \\
& \leq F(z)-\inf _{X} F \leq \hat{\varepsilon}
\end{aligned}
$$

Find an $x_{1} \in S_{0}$, which allows

$$
\begin{gathered}
F\left(x_{1}\right)+\delta_{0} \rho\left(x_{1}, x_{0}\right) \\
\leq \inf _{x \in S_{0}}\left[F(x)+\sigma_{0} \rho\left(x, x_{0}\right)\right]+\sigma_{1} \hat{\varepsilon} / 2 \sigma_{0} .
\end{gathered}
$$

Let

$$
S_{1}=\left\{x \in S_{0} \mid \begin{array}{l}
F(x)+\sum_{k=0}^{1} \sigma_{k} \rho\left(x, x_{k}\right) \\
\leq F\left(x_{1}\right)+\rho_{0}\left(x_{1}, x_{0}\right)
\end{array}\right\}
$$

Assuming that we define $S_{n-1}$ and $x_{n} \in S_{n-1}$, which allow

$$
\begin{gathered}
F\left(x_{n}\right)+\sum_{k=0}^{n-1} \sigma_{k} \rho\left(x_{n}, x_{k}\right) \\
\leq \inf _{x \in S_{n-1}}\left[F(x)+\sum_{k=0}^{n-1} \sigma_{k} \rho\left(x, x_{k}\right)\right]+\hat{\varepsilon} \sigma_{n} / 2^{n} \sigma_{0}
\end{gathered}
$$

Let

$$
S_{n}=\left\{x \in S_{n-1} \mid \begin{array}{l}
F(x)+\sum_{k=0}^{n} \sigma_{k} \rho\left(x, x_{k}\right) \\
\leq F\left(x_{n}\right)+\sum_{k=0}^{n-1} \sigma_{k} \rho\left(x_{n}, x_{k}\right)
\end{array}\right\}
$$

We can obtain that $\left\{S_{n}\right\}_{n=0}^{\infty}$ is a list of nonempty closed set according to Theorem 2.1. So by (7) and (8) we have:

$$
\begin{aligned}
& \sigma_{n} \rho\left(x, x_{n}\right) \\
& \leq {\left[F\left(x_{n}\right)+\sum_{k=0}^{n-1} \sigma_{k} \rho\left(x_{n}, x_{k}\right)\right] } \\
&-\left[F(x)+\sum_{k=0}^{n-1} \sigma_{k} \rho\left(x, x_{k}\right)\right] \\
& \leq {\left[F\left(x_{n}\right)+\sum_{k=0}^{n-1} \sigma_{k} \rho\left(x_{n}, x_{k}\right)\right] } \\
&-\inf _{x \in S_{n-1}}\left[F(x)+\sum_{k=0}^{n-1} \sigma_{k} \rho\left(x, x_{k}\right)\right]
\end{aligned}
$$




$$
\leq \hat{\varepsilon} \sigma_{n} / 2^{n} \sigma_{0} .
$$

Therefore, for any $x \in S_{n}(n=1,2, \cdots)$, we have

$$
\rho\left(x, x_{n}\right) \leq \hat{\varepsilon} / 2^{n} \sigma_{0}
$$

Since $\rho$ is a fuzzy gauge mapping, so by (9) we have

$$
d\left(x, x_{n}\right) \rightarrow 0(n \rightarrow \infty) .
$$

On the other hand, by

$$
S_{n} \subset S_{n-1} \text { and } \operatorname{diam}\left(S_{n}\right) \rightarrow 0,
$$

there exists an unique

$$
y \in \bigcap_{n=0}^{\infty} S_{n},
$$

which allows a sequence $\left\{x_{n}\right\}$ converge to $y \in X$.

Next we will prove that $\left\{x_{n}\right\}$ and $y$ satisfy the conclusion (I)- (III) of Theorem 3.1.

(I) by (4) and

$$
y \in \bigcap_{n=0}^{\infty} S_{n},
$$

it's obviously that $\rho(y, z) \leq \hat{\varepsilon} / \sigma_{0}$;

(II) by (3), (8) and

$$
y \in \bigcap_{n=0}^{\infty} S_{n},
$$

for any natural number $q \geq m$, we have

$$
\begin{gathered}
F\left(x_{0}\right) \geq F\left(x_{m}\right)+\sum_{k=0}^{m-1} \sigma_{k} \rho\left(x_{m}, x_{k}\right) \\
\geq F\left(x_{q}\right)+\sum_{k=0}^{q-1} \sigma_{k} \rho\left(x_{q}, x_{k}\right) \\
\geq F(y)+\sum_{k=0}^{q} \sigma_{k} \rho\left(y, x_{k}\right)
\end{gathered}
$$

Therefore

$$
F\left(x_{0}\right) \geq F(y)+\sum_{k=0}^{q} \sigma_{k} \rho\left(y, x_{k}\right) .
$$

Let $q \rightarrow+\infty$, according to (10), we have

$$
F(z) \geq F(y)+\sum_{k=0}^{+\infty} \sigma_{k} \rho\left(y, x_{k}\right)
$$

(III) Since for any $x \neq y, x \notin \bigcap_{n=0}^{\infty} S_{n}$. There exists natural number $m$ which allows $x \notin S_{m}$. So according to (8) we have

$$
\begin{gathered}
F(x)+\sum_{k=0}^{\infty} \sigma_{k} \rho\left(x, x_{k}\right) \\
\geq F(x)+\sum_{k=0}^{m} \sigma_{k} \rho\left(x, x_{k}\right) \\
\geq F\left(x_{m}\right)+\sum_{k=0}^{m-1} \sigma_{k} \rho\left(x_{m}, x_{k}\right)(\text { 令 } m \rightarrow \infty) .
\end{gathered}
$$

Le t $m \rightarrow+\infty$, according to (11), we have

$$
F(x)+\sum_{k=0}^{\infty} \sigma_{k} \rho\left(x, x_{k}\right) \geq F(y)+\sum_{k=0}^{\infty} \sigma_{k} \rho\left(y, x_{k}\right)
$$

Corollary 3.1. Let $F: R^{n} \rightarrow \mathcal{F}$ be a fuzzy lower semi-continuous bounded mapping. If $F$ satisfy conditions (1)-(3):

(I) $F\left(R^{n}\right)$ is the totally ordered sub set of $\mathcal{F}$ with H-difference;

(II) $\inf _{R^{n}} F \in F\left(R^{n}\right)$ and $\lambda>0, p \geq 1$.

Then for any $\varepsilon>0$ and $z \in R^{n}$, there exists $y \in R^{n}$ which allows:

(I) $\|z-y\| \leq \lambda$;

(II) $F(y) \leq F(z)$;

(III) $F(x)+\frac{\hat{\varepsilon}}{\lambda^{p}}\|x-z\|^{p}$

$$
\geq F(y)+\frac{\hat{\varepsilon}}{\lambda^{p}}\|y-z\|^{p}, \forall x \in R^{n} .
$$

When $F(z) \leq \inf _{R^{n}} F+\hat{\varepsilon}$.

Proof. Let

$$
\rho(x, y)=\|x-z\|^{p} \hat{\varepsilon} / \lambda^{p},
$$

then

$$
\rho: R^{n} \times R^{n} \rightarrow \mathcal{F}_{+}
$$

is a gauge fuzzy mapping.

Take

$$
\sigma_{n}=\frac{1}{2^{n}}(n=0,1,2 \cdots),
$$

then we can easily prove the conclusion of Corollary 3.1 according to Theorem 3.1 . 


\section{Fuzzy Approximate Sum Rule}

Lemma 4.1. Let

$$
F, G: R^{n} \rightarrow \mathcal{F}
$$

be lower semi-continuous fuzzy mapping. If $F\left(R^{n}\right)$ is totally ordered sub set of $\mathcal{F}, G(M) \subset \tilde{R}$ and $G$ is differentiable at point $x_{0}$, we have

$$
\partial(F+G)\left(x_{0}\right)=\partial F\left(x_{0}\right)+\nabla G\left(x_{0}\right) .
$$

Proof. According to Theorem 2.2 (II), we have

$$
\partial F\left(x_{0}\right)+\nabla G\left(x_{0}\right) \subset \partial(F+G)\left(x_{0}\right) .
$$

So we can prove (12) by proving

$$
\partial(F+G)\left(x_{0}\right) \subset \partial F\left(x_{0}\right)+\nabla G\left(x_{0}\right) .
$$

Let

$$
\xi=\left(u_{1}, u_{2}, \cdots, u_{n}\right) \in \partial(F+G)\left(x_{0}\right),
$$

then for any $x \in R^{n}$, we have

$$
(F+G)(x) \geq(F+G)\left(x_{0}\right)+\left\langle\xi, z-x_{0}\right\rangle \text {. }
$$

Therefore for any $\alpha \in[0,1]$, we have

$$
\begin{gathered}
(F+G)(z)_{*}(\alpha) \\
\geq(F+G)\left(x_{0}\right)_{*}(\alpha)+\left\langle\xi_{*}(\alpha), z-x_{0}\right\rangle, \\
(F+G)(z)^{*}(\alpha) \\
\geq(F+G)\left(x_{0}\right)^{*}(\alpha)+\left\langle\xi^{*}(\alpha), z-x_{0}\right\rangle .
\end{gathered}
$$

According to the basic properties of the sub-differential of real valued functions, we have

$$
\begin{aligned}
& \xi_{*}(\alpha) \in \partial(F+G)\left(x_{0}\right)_{*}(\alpha) \\
= & \partial F\left(x_{0}\right)_{*}(\alpha)+\nabla G\left(x_{0}\right)_{*}(\alpha), \\
& \xi^{*}(\alpha) \in \partial(F+G)\left(x_{0}\right)^{*}(\alpha) \\
= & \partial F\left(x_{0}\right)^{*}(\alpha)+\nabla G\left(x_{0}\right)^{*}(\alpha)
\end{aligned}
$$

So there exists

$$
\begin{aligned}
& \xi_{1^{*}}(\alpha) \in \partial F\left(x_{0}\right)_{*}(\alpha) \\
& \xi_{2^{*}}(\alpha) \in \nabla G\left(x_{0}\right)_{*}(\alpha)
\end{aligned}
$$

$$
\begin{aligned}
& \xi_{1}^{*}(\alpha) \in \partial F\left(x_{0}\right)^{*}(\alpha) \\
& \xi_{2}^{*}(\alpha) \in \nabla G\left(x_{0}\right)^{*}(\alpha)
\end{aligned}
$$

Such that

$$
\begin{aligned}
& \xi_{*}(\alpha)=\xi_{1^{*}}(\alpha)+\xi_{2^{*}}(\alpha) \\
& \xi^{*}(\alpha)=\xi_{1}^{*}(\alpha)+\xi_{2}^{*}(\alpha)
\end{aligned}
$$

On the other hand, by $G(M) \subset \tilde{R}$ and $G$ is differentiable at $x_{0}$, we have

$$
\nabla G\left(x_{0}\right)_{*}(\alpha)=\nabla G\left(x_{0}\right)^{*}(\alpha)
$$

and

$$
\nabla G\left(x_{0}\right)=\left\{\xi_{2}\right\}
$$

Therefore for any $\alpha \in[0,1]$, we have

$$
\xi_{2^{*}}(\alpha)=\xi_{2}^{*}(\alpha) \in R^{n}
$$

$\xi_{2^{*}}(\alpha)$ and $\xi_{2}^{*}(\alpha)$ determine a same fuzzy vector $\xi_{2}$. So according to (15), $\xi_{1 *}(\alpha)$ and $\xi_{1}^{*}(\alpha)$ also determine a fuzzy vector $\xi_{1} \in \mathcal{F}^{n}(R)$ for any $\alpha \in[0,1]$. And

$$
\xi=\xi_{1}+\xi_{2}
$$

By (13) and (14), for any $\alpha \in[0,1]$ we have

$$
\begin{aligned}
& F(z)_{*}(\alpha) \geq F\left(x_{0}\right)_{*}(\alpha)+\left\langle\xi_{1^{*}}(\alpha), z-x_{0}\right\rangle, \\
& F(z)^{*}(\alpha) \geq F\left(x_{0}\right)^{*}(\alpha)+\left\langle\xi_{1}^{*}(\alpha), z-x_{0}\right\rangle .
\end{aligned}
$$

Therefore,

$$
F(z) \geq F\left(x_{0}\right)+\left\langle\xi_{1}, z-x_{0}\right\rangle
$$

i.e.

$$
\xi_{1} \in \partial F\left(x_{0}\right)
$$

So

$$
\xi=\xi_{1}+\xi_{2} \in \partial F\left(x_{0}\right)+\nabla G\left(x_{0}\right) .
$$

Theorem 4.2 (Fuzzy Approximate sum rule). Let

$$
F_{j}: R^{n} \rightarrow \mathcal{F}(j=1,2, \cdots, m)
$$

be lower semi-continuous bounded fuzzy mappings. If $F_{j}(j=1,2, \cdots, m)$ satisfy conditions (I)-(II):

(I) $F_{j}\left(R^{n}\right)(j=1,2, \cdots, m)$ is totally ordered subset of $\mathcal{F}$ with H-difference; 
(II) $\inf _{R^{n}} F_{j} \in F_{j}\left(R^{n}\right)(j=1,2, \cdots, m)$.

Then for any $\varepsilon>0$ and $z_{j} \in R^{n}$, there exists $y_{j} \in R^{n}$ and $u_{j} \in \partial F_{j}\left(y_{j}\right)$, which allow:

$$
\hat{0} \in \sum_{j=1}^{m} u_{j}+\hat{\varepsilon} B
$$

when $F\left(z_{j}\right) \leq \inf _{R^{n}} F_{j}+\hat{\varepsilon}$, where $B$ is a unit ball in $R^{n}$.

Proof. Let

$$
F_{j}: R^{n} \rightarrow \mathcal{F}(j=1,2, \cdots, m)
$$

be fuzzy mapping which satisfies the conditions of Theorem 4.2. Then when

$$
\lambda>2 m, \varepsilon>0, z_{j} \in R^{n}
$$

and

$$
F\left(z_{j}\right) \leq \inf _{R^{n}} F+\hat{\varepsilon}
$$

according to 3.1 , there exists $y_{i} \in R^{n}(j=1,2, \cdots, m)$ which allows

$$
\left\|z_{j}-y_{j}\right\|<\lambda
$$

and

$$
F\left(x_{j}\right)+\left\|x_{j}-z_{j}\right\|^{2} \hat{\varepsilon} / \lambda^{2}
$$

obtain the minimum at $x_{j}=y_{j}$. So by Theorem 2.3, we have

$$
\hat{0} \in \partial\left(F_{j}+\left\|x_{j}-z_{j}\right\|^{2} \hat{\varepsilon} / \lambda^{2}\right)\left(y_{j}\right) .
$$

On the other hand, according to Lemma 4.1, we have:

$$
\begin{gathered}
\partial\left(F_{j}+\left(\hat{\varepsilon} / \lambda^{2}\right)\left\|x_{j}-z_{j}\right\|^{2}\right)\left(y_{j}\right) \\
=\partial F_{j}\left(y_{j}\right)+\nabla\left(\left\|x_{j}-z_{j}\right\|^{2} \hat{\varepsilon} / \lambda^{2}\right) \\
=\partial F_{j}\left(y_{j}\right)+\left(2 \hat{\varepsilon} / \lambda^{2}\right)\left(x_{j}-z_{j}\right)(j=1,2, \cdots, m) .
\end{gathered}
$$

According to

$$
\begin{aligned}
& \left\|\sum_{j=1}^{m} \frac{2}{\lambda^{2}}\left(y_{j}-z_{j}\right)\right\| \\
& \leq \frac{2}{\lambda^{2}} \sum_{j=1}^{m}\left\|\left(y_{j}-z_{j}\right)\right\| \\
& \leq \frac{2}{\lambda^{2}} \cdot m \lambda \\
& =\frac{2 m}{\lambda}<1
\end{aligned}
$$

we can obtain

$$
\sum_{j=1}^{m} \frac{2}{\lambda^{2}}\left(y_{j}-z_{j}\right) \in B
$$

Therefore

$$
\hat{0} \in \sum_{j=1}^{m} \partial F_{j}\left(y_{j}\right)+\hat{\varepsilon} B
$$

So there exists $u_{j} \in \partial F_{j}\left(y_{j}\right)(j=1,2, \cdots, m)$, such that $\hat{0} \in \sum_{j=1}^{m} u_{j}+\hat{\varepsilon} B$.

\section{Conclusion}

We are inspired by non-smooth variation principles and try to introduce the skill of non-smooth variation into fuzzy analysis. The basic properties of sub-differential of fuzzy mapping is discussed. A variation principle of fuzzy mapping is proved and applied into the variation problem of fuzzy mappings. Its approximation and rules in sub-differential are given. Studying method in this paper and the conclusion we get may extend a new searching direction and it is hopeful to obtain a new series of conclusion.

\section{Acknowledgements}

This work is supported by the National Science Fund of China (11461052) and the Inner Mongolia Natural Science Foundation of China (2014 MS 0107).

\section{References}

[1] S. S. L. Chang, L. A. Zadeh, On fuzzy mappings and control, IEEE Trans. Syst. Man Cybernet. 2 (1972), 30-34.

[2] M. L. Puri, D. A. Ralescu, Differentials for fuzzy functions, J. Math. Anal. Appl. 91 (1983), 552-558.

[3] M. Panigrahi, G. Panda, S. Nanda, Convex fuzzy mapping with differentiability and its application in fuzzy optimization, European J. Oper. Res., 185 (2008), 47-62.

[4] B. Bede, L. Stefanini, Generalized differentiability of fuzzy-valued functions, Fuzzy Sets Syst. 230 (2013), 119-141. 
[5] C. Zang, X, H. Yuan, E. S. Lee, Duality theory in fuzzy mathematical programming problems with fuzzy coefficients. Comput. Math. Appl., 49 (2005), 1709-1730.

[6] G. X. Wang, C. X. Wu. Directional derivatives and subsifferential of convex fuzzy mappings and application in convex fuzzy programming. Fuzzy Sets and Systems. 138 (2003), 559-591.

[7] Y. E. Bao, B. Dai, Researches on convex extension problems of fuzzy valued functions, J. Math. Comput. Sci., 16 (2015), 239-247.

[8] Y. E. Bao, J. J. Li, A study on the differential and subdifferential of fuzzy mapping and its application problem, J. Nonl. Sci. Appl., 16 (2015), 239-247.
[9] J. M. Borwein, Q. J. Zhu. Techniques of variation analysis. Springer-Verilog, New York, 2005.

[10] F. H. Clarke, Y. S. Ledyaev. Non smooth analysis and control theory. Springer-Verilog, New York, 1998.

[11] T. X. D. Ha. Some variants of the ekeland variational principle for a set-valued mappings. J. Optim. Theory. Appl. 124 (2005), 187-206.

[12] C. X. Wu, C. Wu, The supremum and infimum of the set of fuzzy numbers and its application. J. Math. Anal. Appl. 210 (1997), 499-511.

[13] Y. E. Bao, C. X. Wu. Convexity and semi-continuity of fuzzy mappings. Comput. Math. Appl. 51 (2006), 1809-1816. 